\title{
The magnetic field structure of the central region in M $31^{\star}$
}

\author{
R. Gießübel and R. Beck \\ Max-Planck-Institut für Radioastronomie, Auf dem Hügel 69, 53121 Bonn, Germany \\ e-mail: rbeck@mpifr-bonn.mpg.de
}

Received 6 December 2013 / Accepted 29 July 2014

\begin{abstract}
Context. The Andromeda Galaxy (M31) is the nearest grand-design spiral galaxy. Thus far, most studies in the radio regime concentrated on the $10 \mathrm{kpc}$ ring. The central region of M 31 has significantly different properties than the outer parts: The star formation rate is low, and inclination and position angle are largely different from the outer disk.

Aims. The existing model of the magnetic field in the radial range $6 \leq r \leq 14 \mathrm{kpc}$ is extended to the innermost part $r \leq 0.5 \mathrm{kpc}$ to ultimately achieve a picture of the entire magnetic field in M 31 .

Methods. We combined observations taken with the VLA at $3.6 \mathrm{~cm}$ and $6.2 \mathrm{~cm}$ with data from the Effelsberg 100-m telescope to fill the missing spacings of the synthesis data. The resulting polarization maps were averaged in sectors to analyse the azimuthal behaviour of the polarized intensity (PI), rotation measure (RM), and apparent pitch angle $\left(\phi_{\text {obs }}\right)$. We developed a simplified 3D model for the magnetic field in the central region to explain the azimuthal behaviour of the three observables.

Results. Our 3D model of a quadrupolar or dipolar dynamo field can explain the observed patterns in PI, RM, and $\phi_{\text {obs }}$, while a 2D configuration is not sufficient to explain the azimuthal behaviour. In addition and independent of our model, the RM pattern shows that the spiral magnetic field in the inner $0.5 \mathrm{kpc}$ points outward, which is opposite to that in the outer disk, and has a pitch angle of $\simeq 33^{\circ}$, which is much larger than that of $8^{\circ}-19^{\circ}$ in the outer disk.

Conclusions. The physical conditions in the central region differ significantly from those in the $10 \mathrm{kpc}$ ring. In addition, the orientation of this region with respect to the outer disk is completely different. The opposite magnetic field directions suggest that the central region is decoupled from the outer disk, and we propose that an independent dynamo is active in the central region.
\end{abstract}

Key words. techniques: polarimetric - galaxies: individual: M31 - galaxies: magnetic fields - radio continuum: galaxies polarization

\section{Introduction}

With its distance of only $750 \mathrm{kpc}^{1}$, the Andromeda galaxy (M31) is the nearest grand spiral galaxy and the largest extragalactic object on the sky after the Magellanic Clouds. Its proximity allows us to reach spatial resolutions in this galaxy of less than a kiloparsec with single-dish observations and of less than $100 \mathrm{pc}$ with interferometer data.

The M31 galaxy has been a primary target for single-dish radio telescopes that are able to scan the full angular extent of its emission with high sensitivity. It is thus no surprise that M31 was one of the first external spiral galaxies from which polarized radio continuum emission was detected (Beck et al. 1978). The turbulent and ordered ${ }^{2}$ magnetic field components are concentrated in a torus-like structure at about $10 \mathrm{kpc}$ radius (Beck 1982). This star-forming "ring" is a superposition of several tightly wound spiral arms with small pitch angles seen under a high inclination. The origin of the ring is still a matter of

\footnotetext{
* FITS files of the recombined Stokes IQU images at the two frequencies are only available at the CDS via anonymous ftp to cdsarc.u-strasbg. fr (130.79.128.5) or via http://cdsarc.u-strasbg.fr/viz-bin/qcat?J/A+A/571/A61 $1752 \pm 27 \mathrm{kpc}$ from luminosity of Cepheids (Riess et al. 2012) or $744 \pm 33 \mathrm{kpc}$ using eclipsing binaries (Vilardell et al. 2010).

2 Linearly polarized radio continuum emission traces ordered magnetic fields, which can be either regular (or large-scale) magnetic fields (preserving their direction over large scales) or anisotropic turbulent fields (with multiple field reversals within the telescope beam). To distinguish between these two components observationally, additional Faraday rotation data is needed.
}

discussion. It may either result from an interaction with M 32, one of M 31's satellite galaxies (Gordon et al. 2006; Block et al. 2006), or from a major merger event between two large galaxies that possibly formed M 31 (Hammer et al. 2010; Fouquet et al. 2012). Faraday rotation measures (RM) derived from radio polarization data at two wavelengths showed that the large-scale field of M 31 is regular; it preserves its direction around $360^{\circ}$ in azimuth and across several kiloparsecs in radius (Berkhuijsen et al. 2003; Fletcher et al. 2004).

By now, we know that all observed spiral (and even some irregular) galaxies exhibit ordered magnetic fields with an average field strength of $5 \pm 3 \mu \mathrm{G}$, while the random field generally reaches around three times the strength of the ordered field (Fletcher 2010). In galaxies where Faraday rotation data are available, a significant fraction of the ordered field is regular. The presence of these regular fields can be best explained by the mean-field $\alpha-\Omega$ dynamo theory (Beck et al. 1996; Moss et al. 1998 ) that has recently received support from high-resolution MHD simulations (Gressel et al. 2008; Gent et al. 2013). The mean-field $\alpha-\Omega$ dynamo requires the presence of weak magnetic seed fields and an interplay of turbulence and shear to generate and maintain a regular field. Turbulence is provided by supernova explosions, and shear is a consequence of the differential rotation of the gas in the galactic disk. However, the generation of a regular field in a large galaxy takes billions of years and may not yet have been completed in present-day galaxies (Arshakian et al. 2009). In particular, large-scale field reversals, as relics of the initial seed field, may persist until the present time (Beck et al. 1994; Moss et al. 2012). 
The detection of M31's regular magnetic field was a breakthrough for galactic dynamo theory, and it is still considered the prototype of a dynamo-generated field. The structure of the field of M 31 is unusually simple - an almost purely axisymmetric and plane-parallel field has not yet been found in any other spiral galaxy. According to dynamo models, the toroidal field in the disk should be accompanied by a poloidal field that extends away from the disk, as observed around many edge-on galaxies (Krause 2009) and supported by the distribution of polarized emission in mildly inclined galaxies (Braun et al. 2010). The non-detection of a radio halo around M31 (Gräve et al. 1981; Berkhuijsen et al. 2013; Gießübel et al. 2013) means that either the total field in the halo is weak or that very few cosmic-ray electrons in the $\mathrm{GeV}$ range (responsible for synchrotron emission in the $\mathrm{GHz}$ range) make it to the halo, for instance, due to cooling.

Radio emission from inside the $10 \mathrm{kpc}$ ring of M 31 is restricted to the central region with a radius of about $1.5 \mathrm{kpc}$, leaving a gap between these two regions. The average strength of the total magnetic field in rings around the centre increases from $15 \pm 3 \mu \mathrm{G}$ at $0.2-0.4 \mathrm{kpc}$ radius to $19 \pm 3 \mu \mathrm{G}$ at $0.8-1.0 \mathrm{kpc}$ radius, which is about 3-4 times larger than in the $10 \mathrm{kpc}$ ring (Hoernes et al. 1998). RM from three polarized background sources shining through the gap indicate that the large-scale regular field also exists inside the ring (Han et al. 1998), but it is not illuminated by cosmic-ray electrons. At a frequency of $350 \mathrm{MHz}$, the gap is less pronounced in total power, but also clearly present in polarization (Gießübel et al. 2013). Formation of massive stars and hence sources of cosmic rays are limited to radii $\geq 3 \mathrm{kpc}$ (e.g. Kang et al. 2012). Diffusion of cosmic-ray electrons across the ring is hampered by the regular field because the diffusion coefficient across the field lines is smaller than along the field (Snodin et al. 2006; Yan \& Lazarian 2008; Buffie et al. 2013). The strength of the radio synchrotron emission from the central region raises the question of the origin of cosmic rays in that region. Particle acceleration by magnetic reconnection near the galaxy's centre (Lesch \& Reich 1992) or in shock fronts possibly seen in $\mathrm{H} \alpha$ emission (Jacoby et al. 1985) are under discussion.

Thus far, all polarization studies concentrated on the $10 \mathrm{kpc}$ ring, while very little is known about the magnetic field in the central region. A spiral pattern with a large pitch angle was found in the southern half of the central region (Beck 1982; Berkhuijsen et al. 2003), but the low spatial resolution did not allow to investigate its origin. A connection with the spiral field in the outer ring is hardly possible because the gas disk of the central region has a much smaller inclination than the ring plane (Ciardullo et al. 1988; Melchior \& Combes 2011). It is not known whether the same dynamo can operate in two differently oriented disks or whether two dynamos can operate independently. With our polarization observations and Faraday rotation data with high angular resolution and sensitivity, we can investigate this question.

\section{Observations}

The M 31 galaxy has been observed with the Very Large Array (VLA) at $3.6 \mathrm{~cm}(8.46 \mathrm{GHz})$ on 23 December 2005 and at $6.2 \mathrm{~cm}$ $(4.86 \mathrm{GHz})$ on 26 July 1992 . Both observations were made using the D-Array. The $6.2 \mathrm{~cm}$ data has already been presented in Hoernes (1997) but was reduced again from the raw UV data for this analysis, since the images were contaminated by side-lobes from a bright off-centre source (see below).

The Effelsberg data was taken over several years (1999 (8.35 GHz, $3.6 \mathrm{~cm})$; 2001-2005 (4.85 GHz, $6.2 \mathrm{~cm}))$ and will
Table 1. Observational details.

\begin{tabular}{ll}
\hline \hline Centre position & $\mathrm{RA}=00^{\mathrm{h}} 42^{\mathrm{m}} 46^{\mathrm{s}} .0485$ \\
$(\mathrm{~J} 2000)$ & $\mathrm{Dec}=41^{\circ} 16^{\prime} 11^{\prime \prime} 623$ \\
Distance & $750 \pm 30 \mathrm{kpc}$ \\
Angular resolution & $15^{\prime \prime}$ \\
Along major axis & $15^{\prime \prime} \approx 54.5 \pm 2.2 \mathrm{pc}$ \\
Rms noise ${ }^{a}:$ & \\
$3.6 \mathrm{~cm}$ total power & $\sigma_{\mathrm{t}}=5.5 \mu \mathrm{Jy} / \mathrm{beam}$ \\
$3.6 \mathrm{~cm}$ PI & $\sigma_{\mathrm{PI}}=4.8 \mu \mathrm{Jy} / \mathrm{beam}$ \\
$6.2 \mathrm{~cm}$ total power & $\sigma_{\mathrm{t}}=7.5 \mu \mathrm{Jy} / \mathrm{beam}$ \\
$6.2 \mathrm{~cm}$ PI & $\sigma_{\mathrm{PI}}=6.8 \mu \mathrm{Jy} / \mathrm{beam}$ \\
\hline
\end{tabular}

Notes. ${ }^{(a)}$ The noise values refer to the central area of the primary beam; they increase with distance from the field centre.

be presented in a separate paper (Gießübel et al., in prep.). The $3.6 \mathrm{~cm}$ receiver is a single-horn receiver with a bandwidth of $1.1 \mathrm{GHz}$ centred on $8.35 \mathrm{GHz}$; the $6.2 \mathrm{~cm}$ receiver is a dualhorn receiver with a bandwidth of $0.5 \mathrm{GHz}$ centred on $4.85 \mathrm{GHz}$. Both are installed in the secondary focus of the Effelsberg $100 \mathrm{~m}$ Telescope. Both maps were observed using on-the-fly mapping, alternating in two perpendicular directions.

The observational details are summarized in Table 1.

\section{Data reduction}

Data reduction of the VLA data was made in $\mathrm{AIPS}^{3}$ using the standard tasks and VLACALIB. At both wavelengths, 3C48 was used as a flux calibrator and $3 \mathrm{C} 138$ for polarization calibration (polarization angle $-12^{\circ}$ ). As phase calibrators, 3C19 was observed at $6.2 \mathrm{~cm}$ and $0013+408(\mathrm{~J} 2000)$ at $3.6 \mathrm{~cm}$.

North of the centre, a very bright background source, 37 W115 (Walterbos et al. 1985), is located that is also bright in polarization (e.g. Beck et al. 1989). This source had to be removed from the $6.2 \mathrm{~cm}$ data. The AIPS task PEELR was used to subtract the source from the uv data. At $3.6 \mathrm{~cm}$ the primary beam is much smaller and no obvious side-lobes of $37 \mathrm{~W} 115$ were disturbing the image, so that no peeling was needed for this dataset.

\subsection{Combination with Effelsberg data}

Interferometric data from a synthesis telescope usually suffer from missing zero spacings: the uv plane is never filled entirely, and there is especially a gap between the shortest baselines of the array and zero baseline. The consequence is that a synthesis telescope misses extended emission; it is only sensitive to structures up to $\lambda / D_{\min }$, where $D_{\min }$ is the shortest baseline. The gap in the spatial-frequency domain results in a depression of the response function. In the resulting images this becomes apparent by sources seemingly "sitting in a bowl of negative emission".

The gap in the uv range can be filled using an observation at the same frequency taken with a single-dish telescope that is larger than the shortest spacing of the synthesis telescope. This process is also referred to as feathering. To merge the Effelsberg and VLA data, the AIPS-task IMERG was used. It combines two images in Fourier space. The merged maps are shown in Figs. 1a to $1 \mathrm{~d}$. The maps have a resolution of $15^{\prime \prime}$. Please refer to the image captions and Table 1 for further details.

The central region is seen from below. The northern side is thus closer to the observer than the southern side. Also, the

\footnotetext{
http://www.aips.nrao.edu/
} 


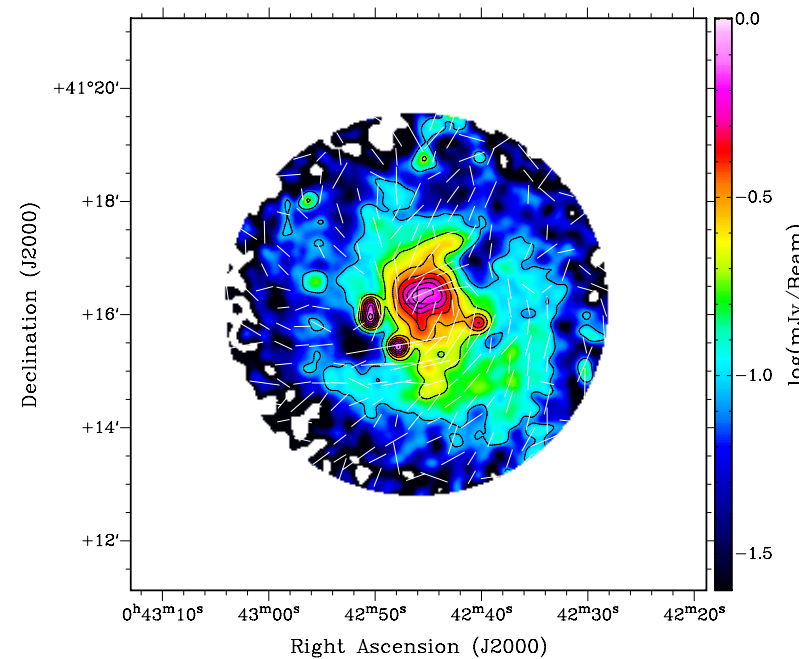

(a) $3.6 \mathrm{~cm}$ total power map

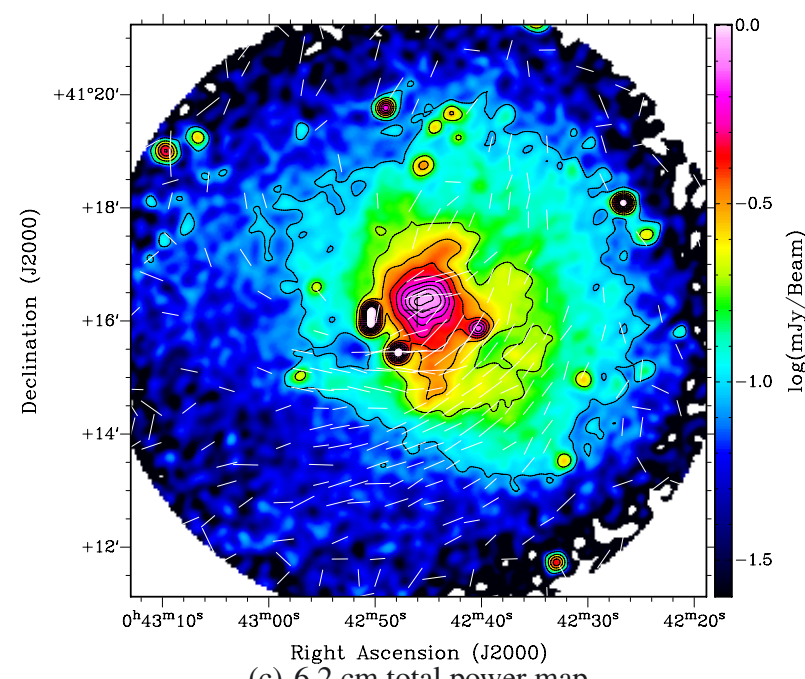

(c) $6.2 \mathrm{~cm}$ total power map

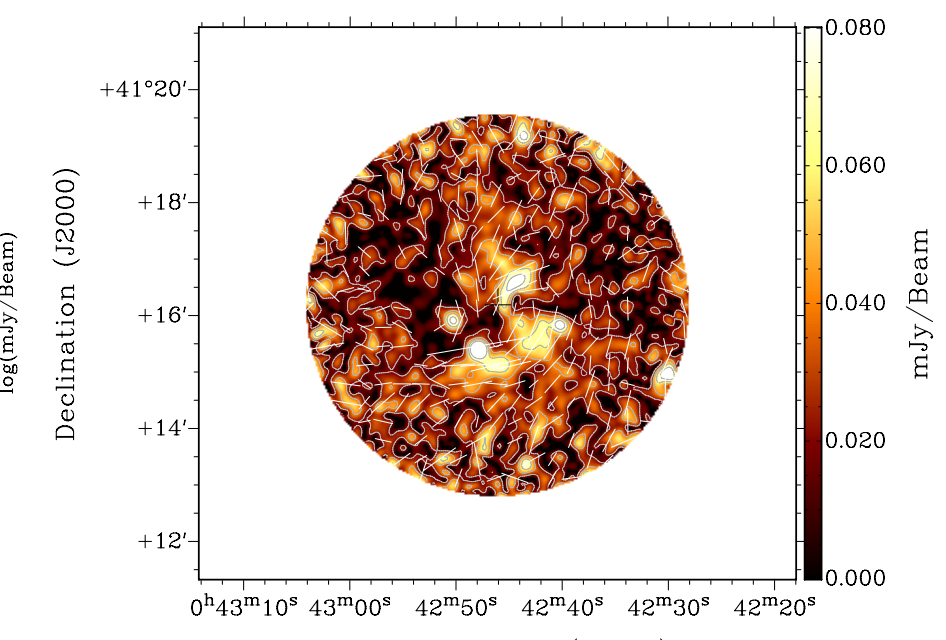

Right Ascension (J2000)

(b) $3.6 \mathrm{~cm}$ polarized intensity map

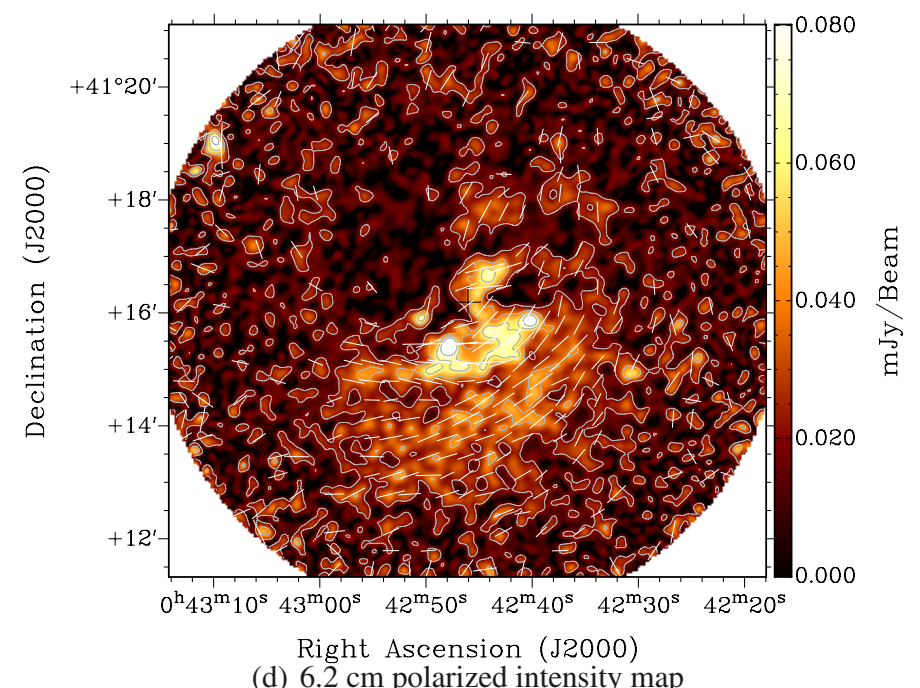

Fig. 1. Total power and polarized intensity maps of the combined VLA and Effelsberg data at $\lambda 3.6 \mathrm{~cm}$ and $\lambda 6.2 \mathrm{~cm}$ at $15^{\prime \prime}$ resolution. The pointing centre of the VLA maps is marked with a black cross. Vectors show the orientation of $\boldsymbol{E}+90^{\circ}$, which are not corrected for Faraday rotation of the foreground. A vector-length of $1^{\prime \prime}$ corresponds to a polarized intensity of $0.01 \mathrm{mJy}$, they are cut off at $3 \sigma_{\mathrm{PI}}$. The range of RM between about $-200 \mathrm{rad} / \mathrm{m}^{2}$ and $+100 \mathrm{rad} / \mathrm{m}^{2}$ (see Fig. 5) corresponds to rotation angles $-15^{\circ}$ to $+7.5^{\circ}$ at $3.6 \mathrm{~cm}$ and $-44^{\circ}$ to $+22^{\circ}$ at $6.2 \mathrm{~cm}$. The depicted $\mathrm{map}$ size is $\sim 9^{\prime} \times 9^{\prime}$. Contours of the total power maps range from 90 to $890 \mu \mathrm{Jy} / \mathrm{beam}$ in steps of $100 \mu \mathrm{Jy} / \mathrm{beam}$ on a logarithmic colour-scale. Contours of the polarized intensity maps range from 20 to $100 \mu \mathrm{Jy} /$ beam in steps of $20 \mu \mathrm{Jy} / \mathrm{beam}$ on a linear colour scale.

north-eastern side is the receding and the south-western the approaching side (e.g. Boulesteix et al. 1987).

The total emission is brightest in the centre. Additionally the southern arm known from $\mathrm{H} \alpha$ emission is clearly visible. Note, however, that the polarized emission is strongest on the inside of the $\mathrm{H} \alpha$ arm and vanishes at the actual position of the arm. Especially at $6.2 \mathrm{~cm}$, the southern arm is significantly depolarized, probably due to Faraday depolarization. The enhancement of emission on the inner side of the arm indicates compression of the magnetic field lines due to weak shocks.

Furthermore, a large part of the emission at $6.2 \mathrm{~cm}$ in the north-east is completely depolarized. The implications of this polarization pattern are discussed in Sect. 7.

\section{Spectral index}

Figure 2 shows the spectral index (SI) map between $3.6 \mathrm{~cm}$ and $6.2 \mathrm{~cm}$. Typical errors for SI are \pm 0.02 in the centre, \pm 0.2 on the southern arm, and \pm 2 towards the edge of the map. There are two features, which can be clearly distinguished in the SI map: the inner $400 \mathrm{pc}$ and the southern arm, known from $\mathrm{H} \alpha$ observations (e.g. Jacoby et al. 1985). The spectral index in the very centre is $-0.40 \pm 0.03$ and steepens outwards where it quickly reaches values of -1.0 and steeper. A similar behaviour was already noted by Hoernes et al. (1998) between $6.2 \mathrm{~cm}$ and $20 \mathrm{~cm}$. There is little $\mathrm{H} \alpha$ emission (Jacoby et al. 1985) and hence little star formation in the centre, so that the flat spectrum is most probably due to synchrotron emission. Hoernes et al. (1998) proposed that the cosmic ray electrons in the inner kiloparsec originate in a mono-energetic source in the centre, which is associated with the central black hole, as proposed for the centre of the Milky Way (Lesch et al. 1988). However, such a source should generate an inverted synchrotron spectrum, which is not the case in M31. We propose that the cosmic-ray sources are related to nuclear activity, such as shocks in gas outflows or inflows, or stellar winds from the cluster of blue stars, as reported by Lauer et al. (2012). 


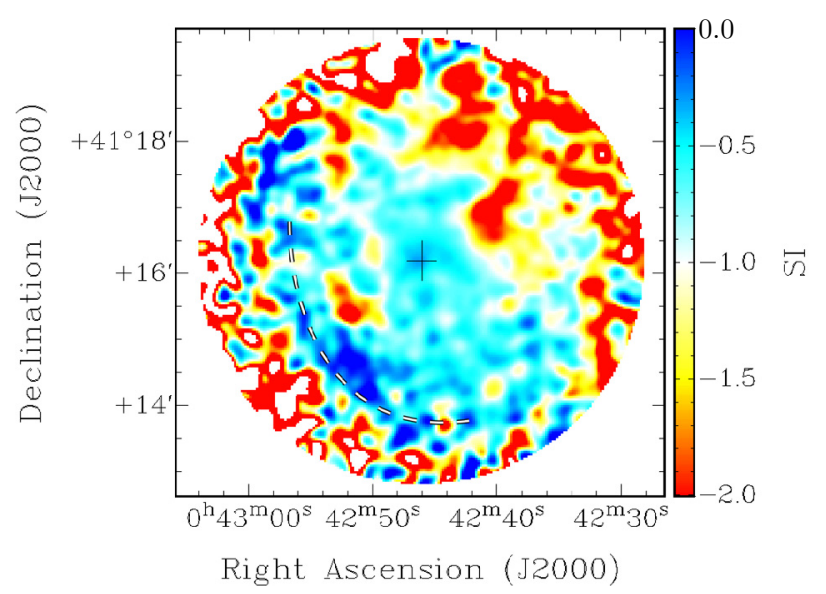

Fig. 2. Spectral index map between $3.6 \mathrm{~cm}$ and $6.2 \mathrm{~cm}$ at $15^{\prime \prime}$ resolution clipped above 0 and below -2 . The dashed line indicates the approximate position of the $\mathrm{H} \alpha$ arm (Jacoby et al. 1985).

The spectral index of the southern arm is again flat with average values between 0.0 and $-0.5( \pm 0.1)$. In the region where it flattens to $0\left(\mathrm{RA}=00^{\mathrm{h}} 42^{\mathrm{m}} 52^{\mathrm{s}} \mathrm{Dec}=+41^{\circ} 14.5\right)$, the polarized intensity is reduced by 10 to $20 \mu \mathrm{Jy} /$ beam on average. Since this position coincides with the $\mathrm{H} \alpha$ arm, we conclude that the emission is dominated by thermal emission and the synchrotron contribution is small in this region. If a significant number of cosmic ray electrons is accelerated by strong shocks or supernova remnants in the southern arm, we would expect a radio spectral index similar to that in normal spiral arms, which around -0.7 . Therefore, the very centre is a more likely source of cosmic ray electrons that we can identify.

\section{Sector analysis of the magnetic field structure}

The inclination $i$ of the plane of the central region and the position angle PA of its major axis differ significantly from the inclination and position angle of the outer disk. While these parameters are very well defined for the $10 \mathrm{kpc}$ ring $\left(i=75^{\circ}, \mathrm{PA}=38^{\circ}\right.$, e.g. Chemin et al. 2009), literature values for the inner kiloparsec vary a lot. Usually an inclination of $i=45^{\circ}$ (Ciardullo et al. 1988 ) is assumed for the nuclear spiral, but the position angle is not well defined due to this mild inclination. Values range from $\mathrm{PA} \approx 38^{\circ}$ like the major axis of the $10 \mathrm{kpc}$-ring (e.g. Boulesteix et al. 1987; Ciardullo et al. 1988) to PA $=48^{\circ}$ (Saglia et al. 2010) and $i=43^{\circ}, \mathrm{PA}=70^{\circ}$ (Melchior \& Combes 2011).

For the following analysis we use $i=43^{\circ}$ and PA $=70^{\circ}$, which is in best accordance with our results. We are, however, not able to further constrain the position angle. We show plots for the other position angles in Sect. 8, where we also address the robustness of our findings with regards to different position angles.

We define four rings with a radial width of $0.1 \mathrm{kpc}$ in the plane of the central disk, starting at $r=0.1 \mathrm{kpc}$ (assuming a distance of $750 \mathrm{kpc}$ to M31). Each ring is divided into sectors with an azimuthal width of $20^{\circ}$ in the disk plane. Figure 3 shows these sectors on top of the $6.2 \mathrm{~cm}$ PI map. The azimuthal angle is counted counter-clockwise, starting at the northern side of the major axis. The central position of the sectors is slightly offset from the pointing centre at RA $=00^{\mathrm{h}} 42^{\mathrm{m}} 44.45$ Dec $=$ $41^{\circ} 16^{\prime} 1^{\prime \prime} 62$ (J2000), so that the sectors are centred on the spiral pattern visible in polarized intensity.

For each sector, we computed the average polarized intensity PI at $6.2 \mathrm{~cm}$, the average RM between $3.6 \mathrm{~cm}$ and $6.2 \mathrm{~cm}$, and

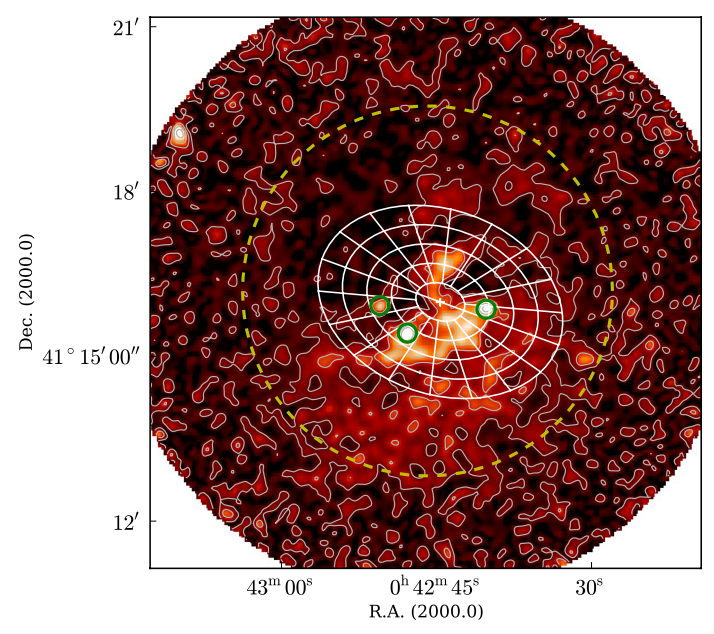

Fig. 3. Sectors for $i=43^{\circ}$ and $\mathrm{PA}=70^{\circ}$ on the $6.2 \mathrm{~cm}$ polarized intensity map. Rings start at $r=0.1 \mathrm{kpc}$ with $\Delta r=0.1 \mathrm{kpc}$, the azimuthal width of the sectors is $20^{\circ}$. They are counted counter-clockwise, starting at the northern side of the major axis. The dashed yellow line shows the size of the primary beam of the $3.6 \mathrm{~cm}$ map. The three strong polarized point sources marked with green circles have been subtracted from the $Q$ and $U$ images. Contours are the same as Fig. 1d.

the pitch angle $\phi_{\text {obs }}$ of the projected average $\boldsymbol{B}$ vectors against the ring orientation as follows:

$P I=\frac{1}{N} \sum_{i}^{N} \sqrt{Q_{i}^{2}+U_{i}^{2}-(1.2 \sigma)^{2}}$,

where $Q_{i}$ and $U_{i}$ is the value at the $i$ th pixel in the $6.2 \mathrm{~cm}$ Stokes $Q$ and Stokes $U$ map, respectively; $\sigma$ is the average noise of the $Q$ and Stokes $U$ maps and $N$ the number of pixels in the sector.

$R M=\frac{\Delta \chi^{2}}{\Delta \lambda^{2}}$

where $\Delta X$ is the difference between the sector averages of the polarization angles at $6.2 \mathrm{~cm}$ and $3.6 \mathrm{~cm}$ calculated as

$\mathcal{X}_{\lambda}=\frac{1}{2} \arctan \frac{\left\langle U_{\lambda}\right\rangle}{\left\langle Q_{\lambda}\right\rangle}$,

here $\left\langle U_{\lambda}\right\rangle$ and $\left\langle Q_{\lambda}\right\rangle$ denote the sector averages of the Stokes $Q$ and Stokes $U$ map at wavelength $\lambda$. The pitch angle is

$\phi=-\theta \pm 90^{\circ}+\arctan \left(\frac{\tan \left(X_{0}-\mathrm{PA}\right)}{\cos i}\right)$,

where $\theta$ is the azimuthal angle of the sector and $X_{0}=X_{\lambda}-$ $R M \lambda^{2} \pm 90^{\circ}$, the intrinsic position angle of the $B$-vector in the plane of the sky. Following the general convention, a positive pitch angle indicates a clockwise winding, and negative pitch angles indicates a counter-clockwise winding spiral. We note that this definition is opposite to that used by Fletcher et al. (2004); we, thus, changed the sign of the cited values according to the definition in this paper.

For a planar magnetic spiral field, the pitch angle represents the pitch angle of the spiral in the plane of the central region. The three strong polarized background sources (marked green in Fig. 3) have been subtracted from the $Q$ and $U$ images before the analysis.

Table 2 lists the weighted average pitch angle of the spiral field in each of the four rings. Although the observed pitch 
Table 2. Average pitch angle of the spiral field in the four rings.

\begin{tabular}{cc}
\hline \hline Mean radius $[\mathrm{kpc}]$ & $\phi\left[^{\circ}\right]$ \\
\hline 0.15 & $30 \pm 5$ \\
0.25 & $33 \pm 3$ \\
0.35 & $33 \pm 3$ \\
0.45 & $33 \pm 3$ \\
\hline
\end{tabular}

angle varies significantly over the azimuthal range, the average still represents the pitch angle of the spiral field component (see Sect. 8).

The sector averages for all rings are shown in Fig. 5. The sector averages of PI clearly show the depolarization of the emission at the northern part of the major axis at $6.2 \mathrm{~cm}$. The sector averages of RM show the characteristic pattern expected for an axis-symmetric spiral (ASS) magnetic field in the disk (e.g. Krause et al. 1989). As mentioned above, the pitch angle varies significantly in a coherent pattern, which is is not expected for a magnetic field confined to a disk.

\section{Large-scale magnetic field reversal in M 31}

The direction of the regular magnetic field is non-ambiguously determined by the observed RM pattern ${ }^{4}$. The entire pattern is shifted by the foreground RM of $-93 \mathrm{rad} \mathrm{m}^{-2}$ (Han et al. 1998; Berkhuijsen et al. 2003; Fletcher et al. 2004), as indicated by the red dashed line in Fig. 5, middle column. If the intrinsic RM of the source is positive, the magnetic field vectors are pointing away from the observer; if the intrinsic RM of the source is negative, the magnetic field vectors are pointing towards the observer. Following Krause et al. (1989), we find that the spiral field in the inner $0.5 \mathrm{kpc}$ is pointing outwards, as depicted in Fig. 4.

Fletcher et al. (2004) studied the regular magnetic field of M 31 using observations at $6 \mathrm{~cm}, 11 \mathrm{~cm}$, and $20 \mathrm{~cm}$. They found that the magnetic field between $6 \mathrm{kpc}$ and $14 \mathrm{kpc}$ is pointing inwards with pitch angles ranging from $\phi=19^{\circ}$ to $\phi=8^{\circ}$, which become smaller with increasing radius. The large-scale fields in the central region and in the $10 \mathrm{kpc}$ ring are thus pointing into opposite directions. The reversal itself happens in the radial range between $1.5 \mathrm{kpc}$ and $6 \mathrm{kpc}$, where the strength of the regular field has a minimum (Beck 1982; Moss et al. 1998).

Radial field reversals can be explained by the mean-field $\alpha-\Omega$ dynamo theory as relics of the initial seed field (Beck et al. 1994; Moss et al. 2012; Moss \& Sokoloff 2013). Fields of opposite polarity are stretched by differential rotation and eventually reconnect. One polarity wins, leaving a coherent axisymmetric disk field as the final configuration (Hanasz et al. 2009). (We note that the sense of the winning polarity is random; it is neither determined by the initial conditions nor by the sense of rotation of the galaxy.) Tidal interactions or continuous injection of turbulent fields may distort or slow down this process (Arshakian et al. 2009; Moss et al. 2012). Ferrière \& Schmitt (2000) showed that a radial field reversal can also be explained in the framework of the $\alpha-\Omega$ dynamo theory by a vertical gradient in the galactic rotation rate. Such a gradient is most likely present in the central disk of M 31 due to the ever-changing inclination and position angles over the entire disk (e.g. Chemin et al. 2009).

As the central region of M 31 is decoupled from the $10 \mathrm{kpc}$ ring due to its entirely different inclination and position angle, reconnection of fields of the inner disk and the ring with different

\footnotetext{
4 We emphasize that the result is purely observational and that no assumptions about the overall field geometry are required.
}

polarities seems impossible. Furthermore, the physical conditions in the central region are different from those outside (i.e. little star formation in the central region but a star-forming ring in the outer part, which coincides with the $10 \mathrm{kpc}$ ring seen in radio emission). For these reasons, we propose that the magnetic fields in the inner $(\$ 4 \mathrm{kpc})$ and outer part $(\gtrsim 4 \mathrm{kpc})$ of M31 are generated independently, as was already suggested by Ruzmaikin \& Shukurov (1981). As the field polarity is a random number, the reconnection process of seed fields has developed differently in the two dynamo-active regions.

The efficiency $\eta$ (given by the dynamo number) and timescale $t_{\mathrm{dyn}}$ of the mean-field $\alpha-\Omega$ dynamo in the thin-disk approximation can be estimated by (Ruzmaikin et al. 1988):

$$
\begin{aligned}
& \eta \simeq 9 h^{2} \Omega|S| / v_{\text {turb }}^{2}, \\
& t_{\text {dyn }} \simeq 3 h /\left((\Omega|S|)^{0.5} l_{\text {turb }}\right) .
\end{aligned}
$$

For a flat rotation curve $\left(v_{\text {rot }}=\right.$ const. $)$ :

$\eta \simeq 9(h / R)^{2}\left(v_{\text {rot }} / v_{\text {turb }}\right)^{2}$,

$t_{\text {dyn }} \simeq 3 h R /\left(v_{\text {rot }} l_{\text {turb }}\right)$,

where $\Omega$ is the angular rotational velocity, $S=R(\partial \Omega / \partial R)$ is the rotational shear, $v_{\text {turb }}$ is the turbulent velocity, $l_{\text {turb }}$ is the basic length scale of turbulence, and $h$ is the scale height of the ionized gas. The turbulent velocity $v_{\text {turb }}$ is known to be mostly independent of the star formation rate (except for starbursts) (Dib et al. 2006), so $v_{\text {turb }}$ can be assumed to be similar in the inner and outer disks of M 31, and $S$ and $h$ are the main driving parameters. The scale height $h$ is probably about three times smaller in the inner disk than that in the outer disk (see below). The rotation curve is roughly flat in the inner disk beyond $1^{\prime} \simeq 0.2 \mathrm{kpc}$ (Boulesteix et al. 1987), as well as in in the outer disk (Corbelli et al. 2010), and the rotation velocities are $200-250 \mathrm{~km} \mathrm{~s}^{-1}$. According to Eqs. (7) and (8), the dynamo efficiency scales with $(h / R)^{2}$ and the dynamo time-scale with $h \cdot R$. With a radius ratio of about $1 / 10$ and a height ratio of about $1 / 3$, the dynamo in the inner disk is about $10 \times$ more efficient and about $30 \times$ faster than that in the outer disk.

In the thin-disk approximation $\alpha-\Omega$ dynamo, the pitch angle of the spiral field can be estimated as $\phi \approx \arctan \left(l_{\text {turb }} / h\right)$ (Beck et al. 1996). Little is known about the variation of $l_{\text {turb }}$ within galaxies, so that we assume $l_{\text {turb }}=$ const. here. The observed ratio of pitch angles in the inner and outer disks is about 3 , and by using $h \approx 1 \mathrm{kpc}$ in the outer disk (Fletcher et al. 2004), this indicates $h \approx 370 \mathrm{pc}$ in the inner disk. With a radius of the inner disk of about $1.5 \mathrm{kpc}$, a scale height of about $370 \mathrm{pc}$ is still consistent with a thin disk.

\section{3D structure of the central magnetic field}

\subsection{Comparison with the Westerbork SINGS survey}

The strong variations of the observed pitch angle with the azimuthal angle suggest that a $2 \mathrm{D}$ configuration is not sufficient to explain the observed sector averages (Fig. 5). Furthermore, the pattern of the observed polarized emission at $6.2 \mathrm{~cm}$ (Fig. 1d) shows a striking resemblance to the polarized intensity images of the spiral galaxies observed in the Westerbork SINGS survey, in which a sample of large, northern galaxies from the Spitzer Infrared Nearby Galaxies Survey (SINGS) were observed with the WSRT in two bands at 1300-1432 MHz and 1631-1763 MHz (Braun et al. 2007): for all moderately inclined galaxies with extended polarized emission, the polarized flux is lowest on the side of the major axis of the projected galaxy disk 


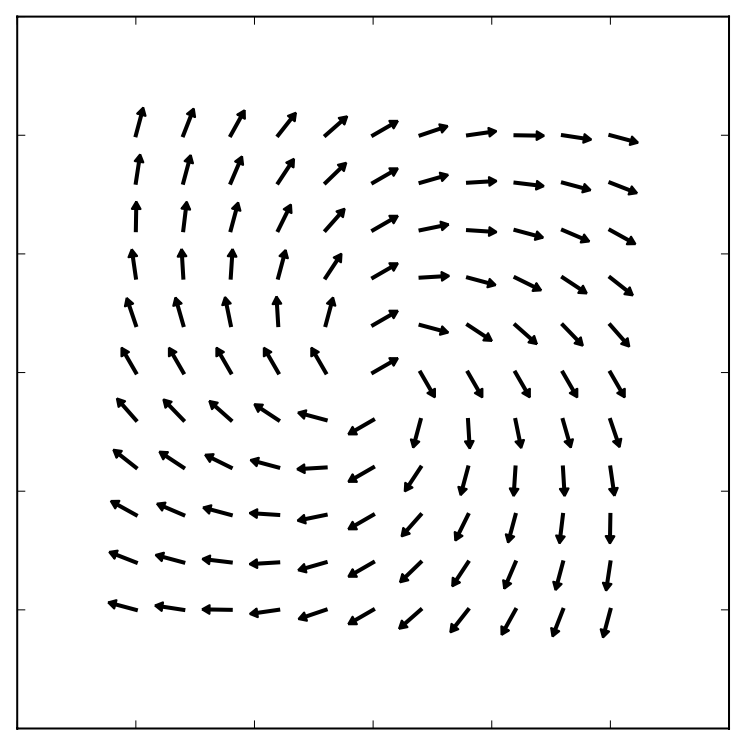

(a)

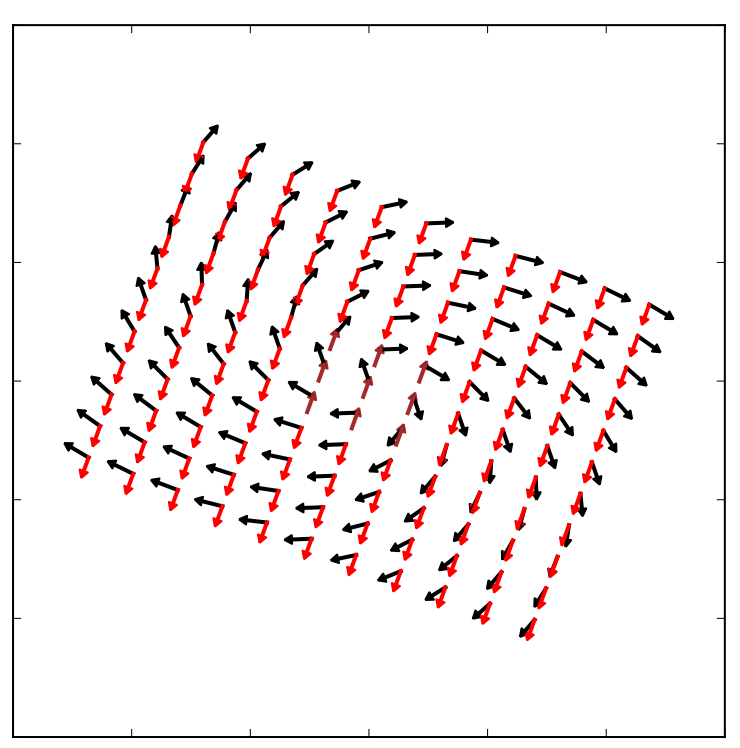

(c)

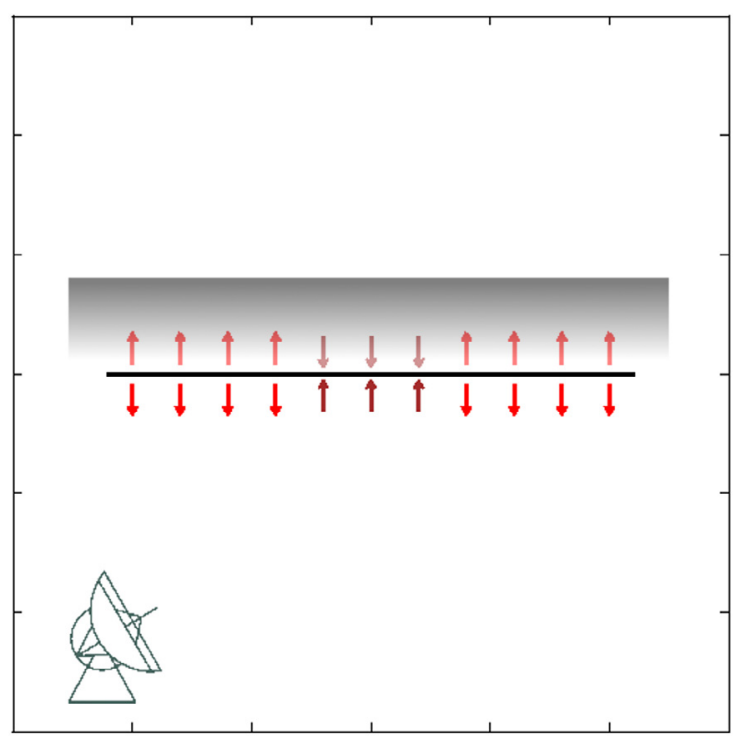

(b)

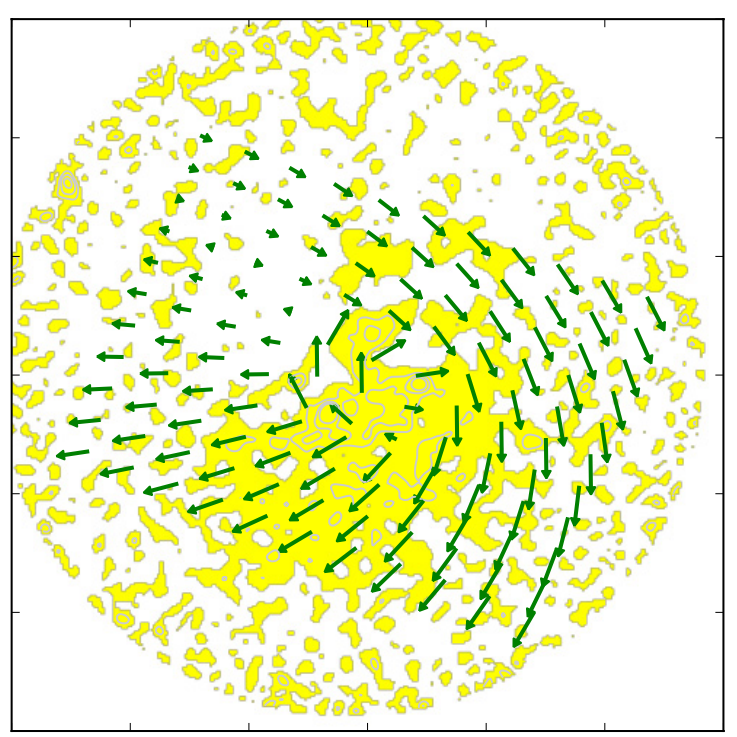

(d)

Fig. 4. Proposed field geometry for the inner $0.7 \mathrm{kpc}$ of the central disk, as seen in projection under different inclinations. The black vectors depict the component in the xy-plane of the disk; the red vectors show the component in the $z$-direction vertical to the disk. The green vectors show the vector addition of both components. a) In-plane component of the vector field seen face-on. The spiral pitch angle is $\phi=33^{\circ}$. b) Out-of-plane "divergence-free" component of the vector field seen edge-on. We observe the field at an inclination. The far side is depolarized at $6.2 \mathrm{~cm}$, so that only the near side is visible to us (indicated by the grey-scale). c) Spiral (black) and vertical (red/brown) components of the field in the sky plane seen under the inclination $i=43^{\circ}$ and the position angle $\mathrm{PA}=70^{\circ}$. Only the near side is visible to us. The kinematically receding side is to the northeast. d) Full vector field of the near side, as seen at $6.2 \mathrm{~cm}$ under the inclination $i=43^{\circ}$ and the position angle PA $=70^{\circ}$. Contours of Fig. $1 \mathrm{~d}$ are underlaid for comparison. For better perceptibility, the lowest contour is filled yellow.

that is receding and highest on the approaching side (Heald et al. 2009; Braun et al. 2010).

In the case of the central region of M31, it is also the receding side that is depolarized (compare Fig. 1d with the velocity field of the central region shown in Fig. 5 of Melchior \& Combes 2011). As there is no indication for enhanced turbulent fields or an enhanced ionized gas density ( $\mathrm{H} \alpha$ emission) on the receding side compared to the approaching side, we propose that the observed pattern in polarized intensity and the asymmetry with respect to the major axis are the result of the 3D field topology projected onto the sky plane and of Faraday effects, as proposed by Braun et al. (2010). They assume that (1) the field structure is of even symmetry (of quadrupolar type); (2) the spiral pattern in the disk follows the optical spiral arms that are trailing with respect to the galaxy rotation; and (3) polarized emission from the far side of the halo is depolarized by Faraday dispersion, so that only polarized emission from the disk and the front side of the halo facing the observer remains visible. Then, in an inclined galaxy disk, the components of the magnetic field of the disk and halo in the sky plane add on the approaching side but partly cancel on the receding side of the major axis.

In the disks of spiral galaxies, sufficiently strong depolarization that fulfils assumption (3) generally occurs at wavelengths of $\geq 20 \mathrm{~cm}$. In the central region of M 31 , however, strong 

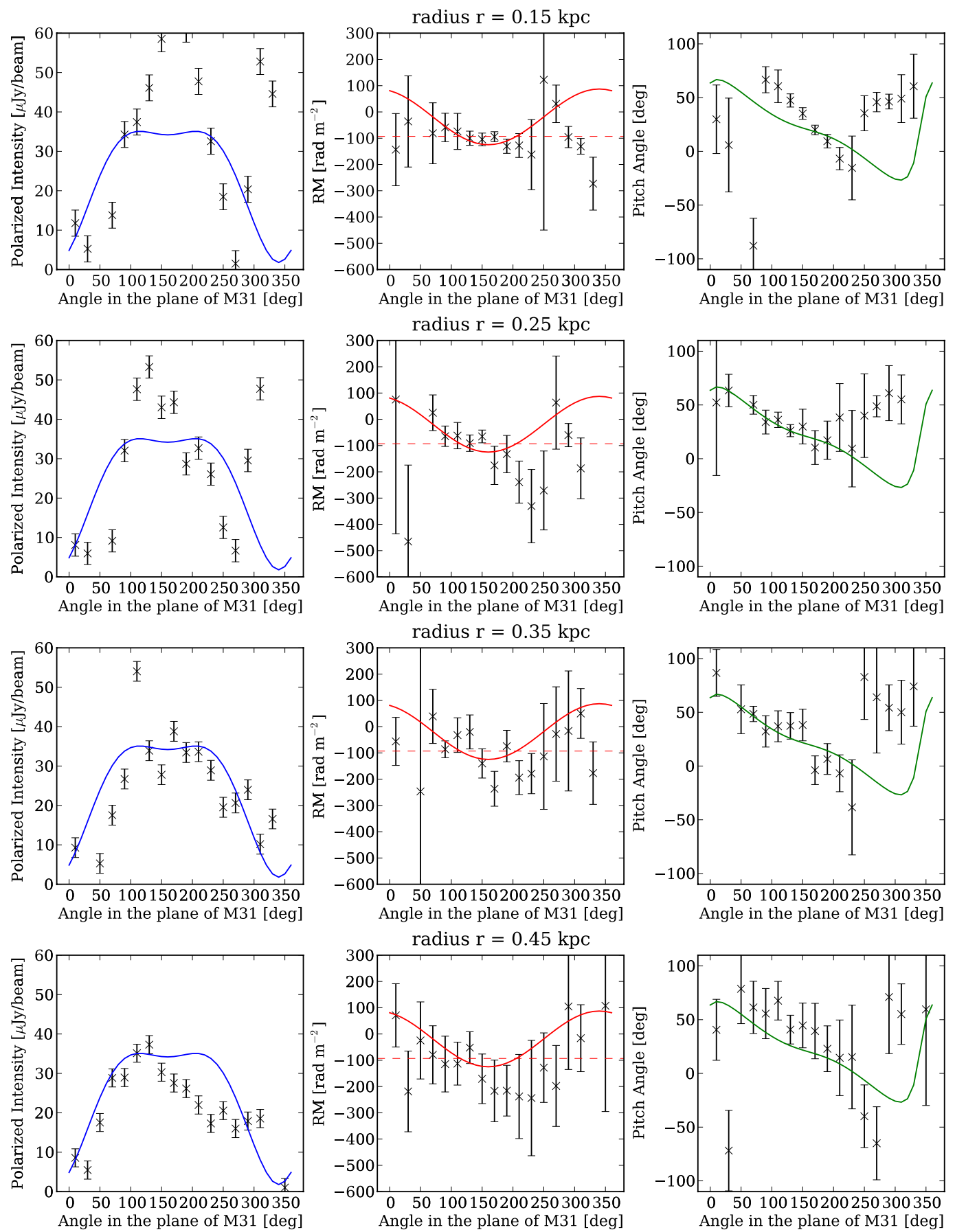

Fig. 5. Points with error bars show (from left to right) the observed values of PI at $6.2 \mathrm{~cm}$, RM between $3.6 \mathrm{~cm}$ and $6.2 \mathrm{~cm}$ and $\phi_{\mathrm{obs}}$ respectively, for the sectors described in Sect. 5. From top to bottom, the results for the rings with mean radius $0.15 \mathrm{kpc}, 0.25 \mathrm{kpc}, 0.35 \mathrm{kpc}$ and $0.45 \mathrm{kpc}$ are presented. The lines are no fits but show the model-output (see Sect. 7.2) using $B_{\mathrm{sp}}=1.0, B_{\mathrm{ver}}=0.7, \phi=30.0^{\circ}, c_{\mathrm{pi}}=20.0, c_{\mathrm{rm}}=150.0, i=43^{\circ}$, and $\mathrm{PA}=70^{\circ}$ for all rings. The red dashed line shows $R M_{\mathrm{fg}}$; the expected rotation measure of the Galactic foreground, by which the model was shifted.

depolarization on the receding side occurs already at $6.2 \mathrm{~cm}$. The polarization degree $p=P I / I$ on the receding side is $p_{6} \simeq 6 \%$ at $6.2 \mathrm{~cm}$ and $p_{3.6} \simeq 20 \%$ at $3.6 \mathrm{~cm}$, while the numbers are similar ( $p_{6} \simeq 17 \%$ and $p_{3.6} \simeq 20 \%$ ) on the approaching side. As the polarized intensity on the receding side is not reduced at $3.6 \mathrm{~cm}$, the far side of the halo is not depolarized at this wavelength.

Depolarization DP by Faraday dispersion can be estimated by

$\mathrm{DP}=\frac{1-\exp \left(-2 \sigma_{\mathrm{RM}}^{2} \lambda^{4}\right)}{2 \sigma_{\mathrm{RM}}^{2} \lambda^{4}}$, where $\sigma_{\mathrm{RM}}$ is the RM dispersion (Sokoloff et al. 1998; Gießübel et al. 2013) that can be expressed as $\sigma_{\mathrm{RM}}=$ $0.81\left\langle n_{\mathrm{e}}\right\rangle B_{\mathrm{r}} d(L /(d f))^{0.5}$ (Beck 2007), where $\left\langle n_{\mathrm{e}}\right\rangle$ is the average thermal electron density of the diffuse ionized gas along the line of sight (in $\mathrm{cm}^{-3}$ ), $B_{\mathrm{r}}$ the random field strength (in $\mu \mathrm{G}$ ), $L$ the pathlength through the thermal gas (in pc), $d$ the turbulent scale (in pc) and $f$ the volume filling factor of the Faraday-rotating gas. Significant depolarization (say, DP $\simeq 0.6$ ) at $6.2 \mathrm{~cm}$ needs $\sigma_{\mathrm{RM}} \simeq 200 \mathrm{rad} \mathrm{m}^{-2}$. This can be achieved by $\left\langle n_{\mathrm{e}}\right\rangle \simeq 0.055 \mathrm{~cm}^{-3}$, $B_{\mathrm{r}} \simeq 20 \mu \mathrm{G}$ (Hoernes et al. 1998), $L=370 / \cos (i) \simeq 500 \mathrm{pc}$ (Sect. 6), $d \simeq 50$ pc and $f \simeq 0.5$. 


\subsection{Our magnetic field model}

To explain the observed polarization pattern, we use a simplified geometrical model, similar to that of Braun et al. (2010), to calculate the magnetic field components along and perpendicular to the line of sight and the apparent pitch angle in the sky plane. The vertical field component has the pattern of a quadrupolar or dipolar ${ }^{5}$ and the horizontal field that of an axisymmetric spiral. Following our observation, the radial field component points outwards (Sect. 6). As a consequence, the vertical component of the poloidal field points towards the plane in the inner region and away from the plane in the outer region. We can decompose this pattern into a log-spiral field in the plane of the disk of the galaxy and a perpendicular component in the vertical direction.

We define the vectors in a fixed coordinate system for a galaxy seen face-on and then rotate the vectors accordingly. We use a right-handed, three-dimensional, Cartesian coordinate system, where the positive $y$-axis points towards the observer. The plane of a galaxy seen face-on thus resides in the $x z$-plane with the positive $z$-axis pointing to the north and the positive $x$-axis pointing to the east.

For any position $\boldsymbol{r}$ in the galaxy at distance $r$ from the centre and at an azimuthal angle $\theta$ counted counter-clockwise from the northern axis, the magnetic field vector is given by

$\boldsymbol{B}=\left(\begin{array}{l}B_{x} \\ B_{y} \\ B_{z}\end{array}\right)=\left(\begin{array}{c}B_{\mathrm{sp}} \cos (\theta+\phi) \\ B_{\mathrm{ver}} \\ B_{\mathrm{sp}} \sin (\theta+\phi)\end{array}\right)$,

where $\phi$ is the pitch angle of the spiral field component and $B_{\text {sp }}$ and $B_{\text {ver }}$ are constants to scale the spiral and vertical field components. We obtain the observed magnetic field vectors $\boldsymbol{B}^{\text {obs }}$ by rotation; the inclination $i$ corresponds to a rotation around the $z$-axis and the position angle, PA, corresponds to a rotation around the $y$-axis,

$\boldsymbol{r}^{\mathrm{obs}}+\boldsymbol{B}^{\mathrm{obs}}=R_{y}(p a) \cdot R_{z}(i) \cdot(\boldsymbol{r}+\boldsymbol{B})$,

where $R_{y}$ and $R_{z}$ are the 3D rotation matrices around the indicated axis by the inserted angle and $\boldsymbol{r}^{\mathrm{obs}}$ is the position seen by the observer. We note that $\boldsymbol{B}^{\text {obs }}$ does not depend on the radius while for an inclined galaxy $B_{x}^{\text {obs }}, B_{y}^{\text {obs }}$ and $B_{z}^{\text {obs }}$ vary with $\theta$ in this simplified model.

Our model does not include a radial dependence of $B_{\text {ver }}$ or $B_{\text {sp }}$, although it can easily be introduced. To compare the model with our data, we use sector averages in rings at fixed radii. Over a large part of the central disk, the radial changes should be small, and only in the innermost and outermost parts of the disk $B_{\text {ver }}$ changes rapidly with $\boldsymbol{r}$. The effects of modifying the different parameters are discussed in Sect. 8.

Figure $4 \mathrm{a}$ sketches the face-on view, where only the logspiral field in the disk-plane is seen. Figure $4 \mathrm{~b}$ shows the vertical component seen edge-on. To mimic a divergence-free field, we introduce a radial dependence of $B_{y}$, so that the vertical field points towards the disk $\left(B_{y}<0\right)$ in the inner disk, while it points away from the disk $\left(B_{y}>0\right)$ in the outer disk. This sharp field reversal is a simplification of a quadrupolar/dipolar field. For comparison with our sector data (Sect. 7.3), we assume that we observe only the outer part of the central disk, where the magnetic field points away from the disk in Fig. 4b. This corresponds to the outer parts in Figs. 11a or 11b.

\footnotetext{
5 With our simple model we can actually not distinguish between a dipolar or quadrupolar-type field (see below). According to the meanfield $\alpha-\Omega$ dynamo model, a quadrupolar-type field is preferred in a thin galactic disk.
}

Figure 4c shows both field components in the sky plane seen under the position angle of $70^{\circ}$ and the inclination of $43^{\circ}$ (see Sect. 5). We observe the disk from below. At $6.2 \mathrm{~cm}$, the far side is depolarized and thus only the vertical component of the near side is seen. Due to this, the symmetry across the mid-plane is unknown, and we cannot distinguish between a dipolar and a quadrupolar field configuration. The observed magnetic field is the vector addition of the spiral component in the disk and the vertical component (we note that both are the components of the same quadrupolar/dipolar-type dynamo field), which is shown in Fig. $4 \mathrm{~d}$. The length of the vectors corresponds to the projected magnetic field strength in the sky plane and thus to the observed polarized intensity. The similarity to the observed polarized intensity at $6.2 \mathrm{~cm}$ (shown in contours) is obvious and shows that our model is valid in a qualitative way.

\subsection{Comparison with our sector data}

The observed polarized intensity is proportional to the projection of $\boldsymbol{B}^{\text {obs }}$ to the $x z$-plane; the observed RM is proportional to the $y$-component of $\boldsymbol{B}^{\text {obs }}$, and the observed apparent pitch angle can be calculated from the projection of $\boldsymbol{B}^{\text {obs }}$ to the $x z$-plane:

$$
\begin{aligned}
& P I=c_{\mathrm{pi}}\left(\sqrt{\left(B_{x}^{\mathrm{obs}}\right)^{2}+\left(B_{z}^{\mathrm{obs}}\right)^{2}}\right)^{1-\alpha} \\
& R M=c_{\mathrm{rm}} B_{y}^{\text {obs }}+R M_{\mathrm{fg}} \\
& \phi_{\mathrm{obs}}=-\theta+\arctan \left(\frac{B_{z}^{\mathrm{obs}}}{B_{x}^{\mathrm{obs}}}\right)-\mathrm{PA},
\end{aligned}
$$

where $\alpha=-0.7$ is the spectral index (recall that the synchrotron intensity $I_{v} \propto B_{\perp}{ }^{1-\alpha} v^{\alpha}$ ). The variable $R M_{\mathrm{fg}}$ is the rotation measure contribution from the foreground, which shifts the observed RM pattern by a constant offset (see Sect. 6).

The variables $c_{\mathrm{pi}}$ and $c_{\mathrm{rm}}$ are proportionality constants used to scale the amplitude of the model output. In a physical context they hold information about the number density of relativistic particles per energy interval and the thermal electron density and path length along the line of sight. In this qualitative analysis we are only interested in the geometry of the magnetic field, which is fully determined by the proportionality between the observed quantities and the projected magnetic field components, not by their absolute quantities.

In Fig. 5 we used $B_{\mathrm{sp}}=1.0, B_{\mathrm{ver}}=0.7, \phi=30.0^{\circ}, c_{\mathrm{pi}}=20.0$, $c_{\mathrm{rm}}=150.0, i=43^{\circ}$, and $\mathrm{PA}=70^{\circ}$ and plot the model output on top of the corresponding sector data (Sect. 5) for PI, RM, and $\phi_{\text {obs }}$. We note that we use the same model for all four rings (so a deviation for the innermost rings is expected) and we did not fit any parameters. We use four rings with a radial width of $0.1 \mathrm{kpc}$ starting at $0.1 \mathrm{kpc}$ radius from the centre. Our geometrical model describes the behaviour of all three observables well.

\section{Robustness of the results}

In Figs. 6 to 9, we show the resulting model outputs for different parameters (see Eqs. (12) to (14)), for comparison with Fig. 5. Only one parameter is varied at a time, the remaining parameters are fixed to $i=43^{\circ}, \phi=33^{\circ}, B_{\mathrm{sp}}=B_{\mathrm{ver}}=1.0$. The scaling parameters $c_{p i}, c_{\mathrm{rm}}$ and $R M_{\mathrm{fg}}$ are not used (i.e. $c_{\mathrm{pi}}=c_{\mathrm{rm}}=1$, $\left.R M_{\mathrm{fg}}=0\right)$.

Figure 6 shows the behaviour for different inclinations. The variation in polarized intensity increases with inclination and starts to show two peaks when exceeding $40^{\circ}$. The variations 
R. Gießübel and R. Beck: The magnetic field structure of the central region in M 31
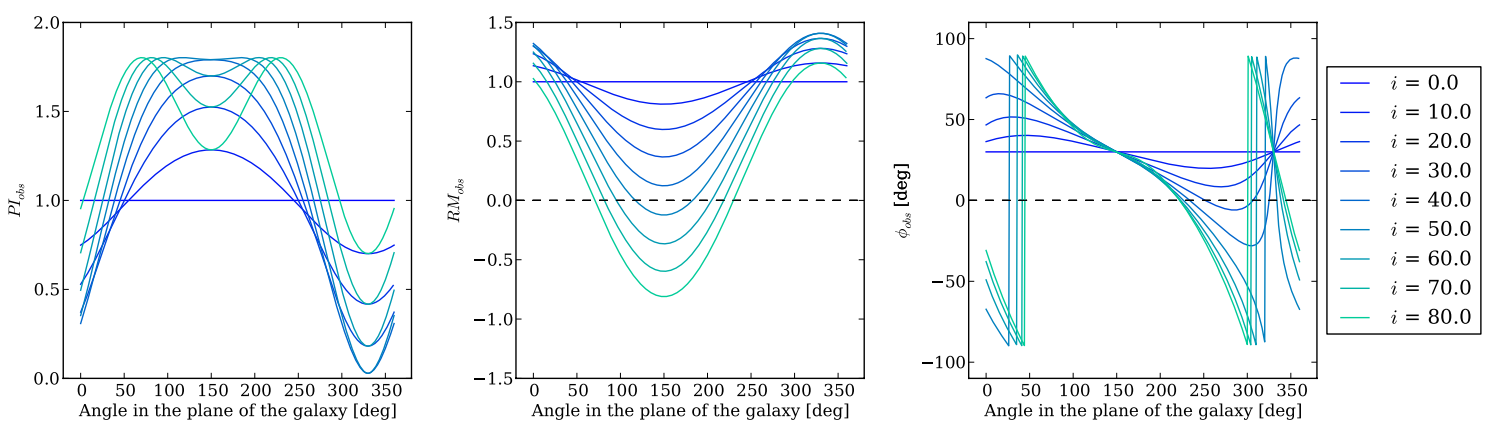

Fig. 6. Model output (from left to right: polarized intensity, rotation measure, and observable pitch angle as a function of azimuthal angle) for different inclinations $i$.
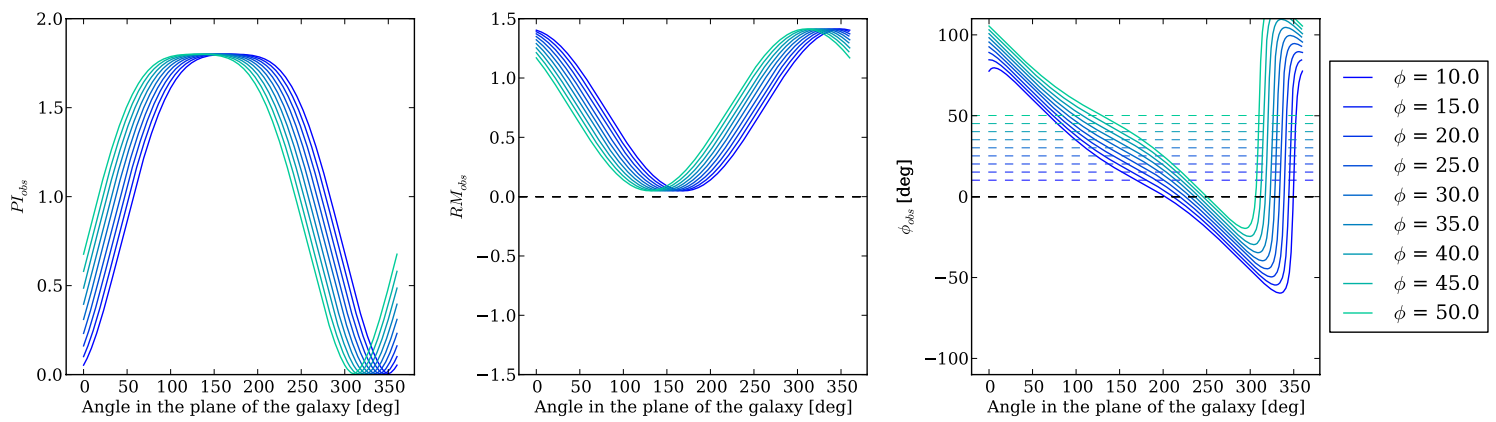

Fig. 7. Model output (from left to right: polarized intensity, rotation measure, and observable pitch angle as a function of azimuthal angle) for different pitch angles $\phi$ of the spiral field component. The dashed horizontal lines indicate the average values of $\phi_{\text {obs }}$.
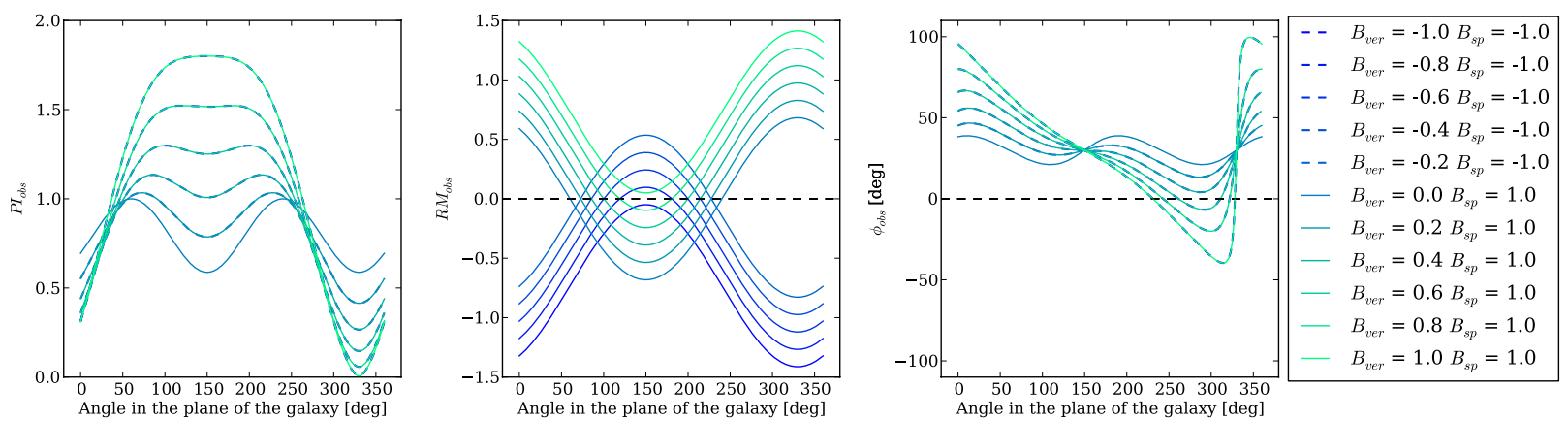

Fig. 8. Model output (from left to right: polarized intensity, rotation measure, and observable pitch angle as a function of azimuthal angle) for different strengths of the vertical field component $B_{\text {ver. }}$.
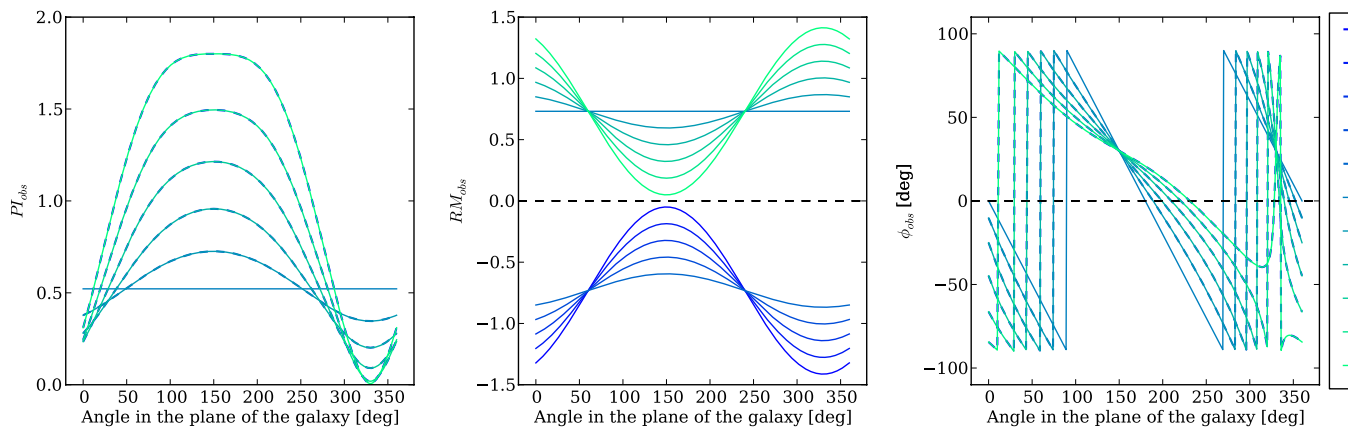

\begin{tabular}{ll|}
-- & $B_{s p}=-1.0 B_{v e r}=-1.0$ \\
-- & $B_{s p}=-0.8 B_{v e r}=-1.0$ \\
-- & $B_{s p}=-0.6 B_{v e r}=-1.0$ \\
-- & $B_{s p}=-0.4 B_{v e r}=-1.0$ \\
-- & $B_{s p}=-0.2 B_{v e r}=-1.0$ \\
- & $B_{s p}=0.0 B_{v e r}=1.0$ \\
- & $B_{s p}=0.2 B_{v e r}=1.0$ \\
- & $B_{s p}=0.4 B_{v e r}=1.0$ \\
- & $B_{s p}=0.6 B_{v e r}=1.0$ \\
- & $B_{s p}=0.8 B_{v e r}=1.0$ \\
- & $B_{s p}=1.0 B_{v e r}=1.0$
\end{tabular}

Fig. 9. Model output (from left to right: polarized intensity, rotation measure, and observable pitch angle as a function of azimuthal angle) for different strengths of the spiral field component $B_{\mathrm{sp}}$.

in $\mathrm{RM}$ and $\phi_{\mathrm{obs}}$ also increase with inclination, so that a minimum inclination of $20^{\circ}$ can be safely assumed from our data. At least for the outer three rings, the data does not indicate a clear double-peak structure for PI and the observed pitch angle does not significantly wrap around $\pm 90^{\circ}$. The behaviour of the observed RM also excludes inclinations larger than $50^{\circ}$, since the RM does not change too rapidly with the azimuthal angle.
The assumed inclination of $i=43^{\circ}$ taken from the literature is consistent with our data but cannot be further constrained.

Varying the pitch angle of the spiral component $\phi$ does not change the observable patterns of PI, RM and $\phi_{\text {obs }}$ (Fig. 7) but only causes a shift in azimuthal angle and $\phi_{\text {obs. }}$. The mean of the observable pitch angle over the entire azimuthal range represents the pitch angle of the spiral field. 

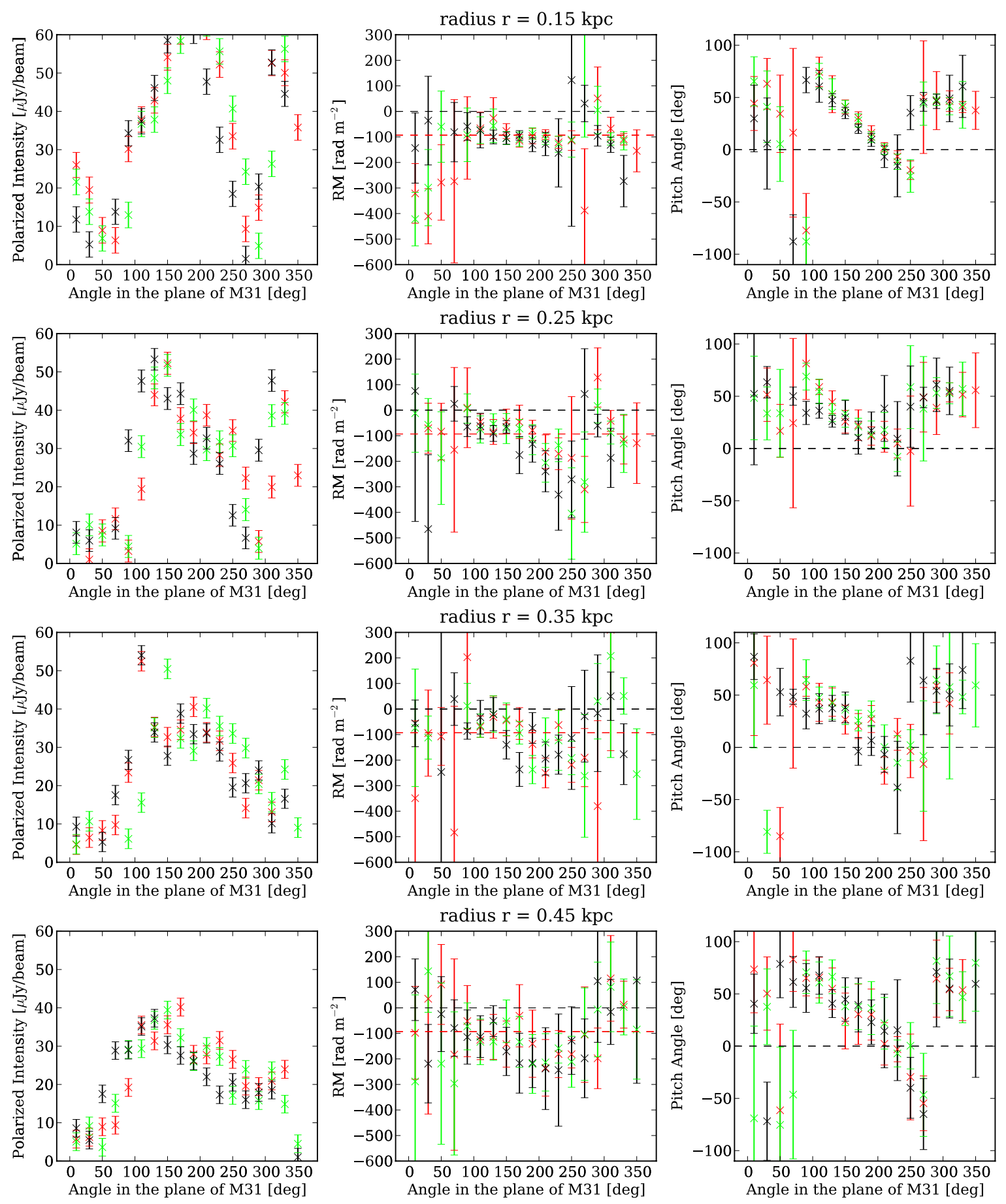

Fig. 10. Result for different position angles of ellipses defining our sectors. Red: PA $=38^{\circ}$ (e.g. Boulesteix et al. 1987; Ciardullo et al. 1988), green: $\mathrm{PA}=48^{\circ}$ (Saglia et al. 2010), and black: PA $=70^{\circ}$ (Melchior $\&$ Combes 2011).

The spiral pitch angle is thus well determined by our observations.

Figures 8 and 9 illustrate that the direction of the field vectors is very well constrained by the three observables. The spiral field component $B_{\mathrm{sp}}$ must be positive (i.e. the field must point outwards) to explain the observed RM pattern. The relative strength of $B_{\mathrm{sp}}$ and $B_{\mathrm{ver}}$ is best constrained by $\phi_{\mathrm{obs}}$. As expected for a quadrupolar or dipolar field configuration, our data is most consistent with $B_{\mathrm{sp}} \geq B_{\text {ver }}$ and $B_{\text {ver }}>0$.

We note that the general directions of $B_{\mathrm{sp}}$ and $B_{\mathrm{ver}}$ cannot be chosen freely for a fixed radius, since the radial direction is coupled to the direction of the halo field. As illustrated in Fig. 11, if $B_{\text {ver }}$ is directed outwards in the outer disk, the radial component has to point outwards as well and vice versa. This is obvious for a quadrupolar field. In a dipolar field, the radial component changes direction across the mid-plane. Our simple model can thus not distinguish between a dipolar or quadrupolar field configuration.

A combination of $B_{\mathrm{sp}}$ and $B_{\mathrm{ver}}$ with opposite sign is only possible in the innermost part, where the field lines return to the disk. If only $B_{\text {ver }}$ changes its sign, the patterns of PI and $\phi_{\text {obs }}$ are shifted by $180^{\circ}$ in azimuthal angle (which at least for PI can be seen in Fig. 4). The deviations from the model in the innermost ring with radius $0.15 \mathrm{kpc}$ (Fig. 5), especially for PI, 


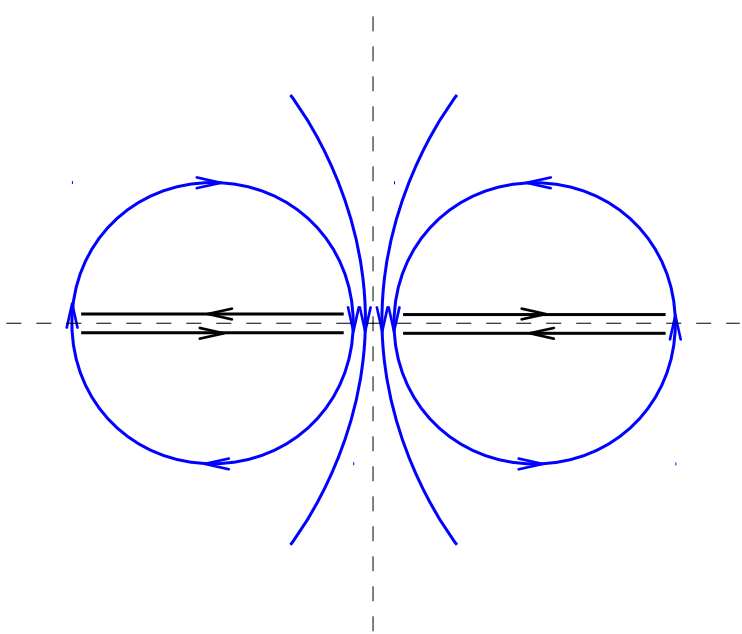

(a)

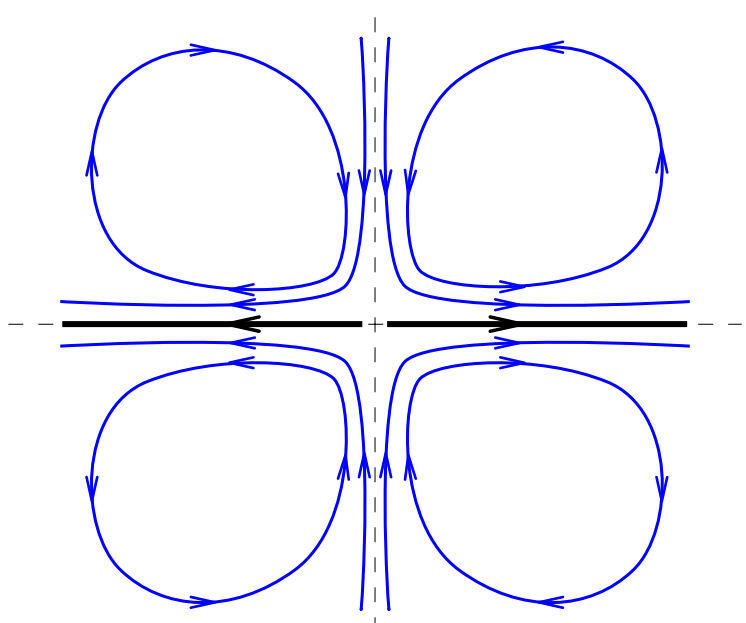

(b)

Fig. 11. Cartoon to visualize that the general directions of $B_{\mathrm{sp}}$ and $B_{\text {ver }}$ are coupled. The poloidal component (blue) of a dipolar a) and a quadrupolar b) field. In addition, we show the direction of the radial field component (black). We note that for the dipolar field the radial component above and below the mid-plane are oppositely directed. The directions of the vertical and spiral components of our model are thus coupled.

give an indication for the closing of the field lines in the centre, but the number of independent resolution elements per sector does not allow a verification using the innermost part.

In Fig. 10, we plot the observed data using different position angles found in the literature to define our sectors, as this might affect the observed patterns. The general behaviour of PI, RM and $\phi_{\text {obs }}$ does not change for different position angles. Within the errors, it is not possible to further constrain the inclination nor the position angle of the central region with the present data.

\section{Conclusions}

(1) Our radio polarization maps of the central region of M31 at $3.6 \mathrm{~cm}$ and $6.2 \mathrm{~cm}$, as obtained from combined data from the VLA and Effelsberg telescopes, reveal a spiral field with a pitch angle of $\sim 33^{\circ}$, which is much larger than the spiral pitch angle of $8^{\circ}-19^{\circ}$ in the outer disk. This difference can be explained in terms of the $\alpha-\Omega$ dynamo theory by the different scale heights $(\approx 0.37 \mathrm{kpc}$ and $\approx 1 \mathrm{kpc}$, respectively) of the ionized gas.

(2) The central region is partly Faraday-thick at $6.2 \mathrm{~cm}$, so that only emission from the near side is observed. The polarization degree at $6.2 \mathrm{~cm}$ is small around the north-eastern major axis of the projected disk and large on the south-western side.

(3) A three-dimensional field configuration can explain the observed patterns in polarized intensity, rotation measure and polarization angle in the inner kiloparsec of M31. With the present data and our simple model we can, however, not distinguish between a pattern resulting from the central part of an ASS-quadrupolar or ASS-dipolar configuration (which would both appear helical in the innermost part).

(4) The magnetic field vectors of the central magnetic field spiral in the radial range $0.2-0.5 \mathrm{kpc}$ point outwards. This is a safe observational result from the rotation measures between $3.6 \mathrm{~cm}$ and $6.2 \mathrm{~cm}$. As the magnetic field in the $10 \mathrm{kpc}$ ring is known to be directed inwards (Fletcher et al. 2004), the inner and outer fields point into opposite directions. This result is not affected by the uncertainty in the orientation of the central disk. As the inner region and the outer disk are physically decoupled, we propose that two independent dynamo-active regions have generated these field patterns, as was already suggested by Ruzmaikin \& Shukurov (1981), where the inner dynamo is more efficient and faster than the outer one.

Acknowledgements. Based on observations with the $100 \mathrm{~m}$ telescope of the MPIfR (Max-Planck-Institut für Radioastronomie) at Effelsberg. All model calculations were made in iPython (Pérez \& Granger 2007) using Matplotlib (Hunter 2007). The National Radio Astronomy Observatory is a facility of the National Science Foundation operated under cooperative agreement by Associated Universities, Inc. We thank Katia Ferrière, Dmitry Sokoloff, Elly Berkhuijsen and our referee Andrew Fletcher for very helpful discussions and comments. RB acknowledges support from DFG FOR1254. The presented results and images have in part been published in Gießübel (2012).

\section{References}

Arshakian, T. G., Beck, R., Krause, M., \& Sokoloff, D. 2009, A\&A, 494, 21

Beck, R. 1982, A\&A, 106, 121

Beck, R. 2007, A\&A, 470, 539

Beck, R., Berkhuijsen, E. M., \& Wielebinski, R. 1978, A\&A, 68, L27

Beck, R., Loiseau, N., Hummel, E., et al. 1989, A\&A, 222, 58

Beck, R., Poezd, A. D., Shukurov, A., \& Sokoloff, D. D. 1994, A\&A, 289, 94

Beck, R., Brandenburg, A., Moss, D., Shukurov, A., \& Sokoloff, D. 1996, ARA\&A, 34, 155

Berkhuijsen, E. M., Beck, R., \& Hoernes, P. 2003, A\&A, 398, 937

Berkhuijsen, E. M., Beck, R., \& Tabatabaei, F. S. 2013, MNRAS, 435, 1598

Block, D. L., Bournaud, F., Combes, F., et al. 2006, Nature, 443, 832

Boulesteix, J., Georgelin, Y. P., Lecoarer, E., Marcelin, M., \& Monnet, G. 1987, A\&A, 178, 91

Braun, R., Oosterloo, T. A., Morganti, R., Klein, U., \& Beck, R. 2007, A\&A, 461,455

Braun, R., Heald, G., \& Beck, R. 2010, A\&A, 514, A42

Buffie, K., Heesen, V., \& Shalchi, A. 2013, ApJ, 764, 37

Chemin, L., Carignan, C., \& Foster, T. 2009, ApJ, 705, 1395

Ciardullo, R., Rubin, V. C., Ford, Jr., W. K., Jacoby, G. H., \& Ford, H. C. 1988, AJ, 95, 438

Corbelli, E., Lorenzoni, S., Walterbos, R., Braun, R., \& Thilker, D. 2010, A\&A, 511, A89

Dib, S., Bell, E., \& Burkert, A. 2006, ApJ, 638, 797

Ferrière, K., \& Schmitt, D. 2000, A\&A, 358, 125

Fletcher, A. 2010, in ASP Conf. Ser. 438, eds. R. Kothes, T. L. Landecker, \& A. G. Willis, 197

Fletcher, A., Berkhuijsen, E. M., Beck, R., \& Shukurov, A. 2004, A\&A, 414, 53

Fouquet, S., Hammer, F., Yang, Y.-B., et al. 2012, EPJ Web Conf., 19, 1007

Gent, F. A., Shukurov, A., Sarson, G. R., Fletcher, A., \& Mantere, M. J. 2013, MNRAS, 430, L40

Gießübel, R. 2012, Ph.D. dissertation, Universität zu Köln, Cuvillier Verlag Göttingen, Germany

Gießübel, R., Heald, G., Beck, R., \& Arshakian, T. G. 2013, A\&A, 559, A27 
Gordon, K. D., Bailin, J., Engelbracht, C. W., et al. 2006, ApJ, 638, L87 Gräve, R., Emerson, D. T., \& Wielebinski, R. 1981, A\&A, 98, 260 Gressel, O., Elstner, D., Ziegler, U., \& Rüdiger, G. 2008, A\&A, 486, L35 Hammer, F., Yang, Y. B., Wang, J. L., et al. 2010, ApJ, 725, 542 Han, J. L., Beck, R., \& Berkhuijsen, E. M. 1998, A\&A, 335, 1117 Hanasz, M., Wóltański, D., \& Kowalik, K. 2009, ApJ, 706, L155 Heald, G., Braun, R., \& Edmonds, R. 2009, A\&A, 503, 409 Hoernes, P. 1997, Ph.D. Thesis, Rheinische Friedrich-Wilhelms-Universität zu Bonn, Germany

Hoernes, P., Beck, R., \& Berkhuijsen, E. M. 1998, in The Central Regions of the Galaxy and Galaxies, ed. Y. Sofue, IAU Symp., 184, 351

Hunter, J. D. 2007, Comput. Sci. Eng., 9, 90

Jacoby, G. H., Ford, H., \& Ciardullo, R. 1985, ApJ, 290, 136

Kang, Y., Rey, S.-C., Bianchi, L., et al. 2012, ApJS, 199, 37

Krause, M. 2009, Rev. Mex. Astron. Astrofis. Conf. Ser., 36, 25

Krause, M., Hummel, E., \& Beck, R. 1989, A\&A, 217, 4

Lauer, T. R., Bender, R., Kormendy, J., Rosenfield, P., \& Green, R. F. 2012, ApJ, 745,121

Lesch, H., \& Reich, W. 1992, A\&A, 264, 493
Lesch, H., Schlickeiser, R., \& Crusius, A. 1988, A\&A, 200, L9 Melchior, A.-L., \& Combes, F. 2011, A\&A, 536, A52

Moss, D., \& Sokoloff, D. 2013, Geophys. Astrophys. Fluid Dynamics, 107, 497 Moss, D., Shukurov, A., Sokoloff, D. D., Berkhuijsen, E. M., \& Beck, R. 1998, A\&A, 335, 500

Moss, D., Stepanov, R., Arshakian, T. G., et al. 2012, A\&A, 537, A68

Pérez, F., \& Granger, B. E. 2007, Comput. Sci. Eng., 9, 21

Riess, A. G., Fliri, J., \& Valls-Gabaud, D. 2012, ApJ, 745, 156

Ruzmaikin, A. A., \& Shukurov, A. M. 1981, Soviet Ast., 25, 553

Ruzmaikin, A. A., Sokolov, D. D., \& Shukurov, A. M. 1988, Magnetic fields of galaxies, Astrophys. Space Sci. Lib., 133

Saglia, R. P., Fabricius, M., Bender, R., et al. 2010, A\&A, 509, A61

Snodin, A. P., Brandenburg, A., Mee, A. J., \& Shukurov, A. 2006, MNRAS, 373, 643

Sokoloff, D. D., Bykov, A. A., Shukurov, A., et al. 1998, MNRAS, 299, 189

Vilardell, F., Ribas, I., Jordi, C., Fitzpatrick, E. L., \& Guinan, E. F. 2010, A\&A, 509, A70

Walterbos, R. A. M., Brinks, E., \& Shane, W. W. 1985, A\&AS, 61, 451

Yan, H., \& Lazarian, A. 2008, ApJ, 673, 942 